University of Wollongong

Research Online

Faculty of Engineering and Information

Faculty of Engineering and Information

Sciences - Papers: Part B

Sciences

2020

\title{
A Novel Multifeature Based On-Site Calibration Method for LiDAR-IMU System
}

Wanli Liu

Zhixiong $\mathrm{Li}$

University of Wollongong, lizhixio@uow.edu.au

Reza Malekian

Miguel Sotelo

Zhenjun Ma

University of Wollongong, zhenjun@uow.edu.au

See next page for additional authors

Follow this and additional works at: https://ro.uow.edu.au/eispapers1

Part of the Engineering Commons, and the Science and Technology Studies Commons

Research Online is the open access institutional repository for the University of Wollongong. For further information contact the UOW Library: research-pubs@uow.edu.au 


\title{
A Novel Multifeature Based On-Site Calibration Method for LiDAR-IMU System
}

\begin{abstract}
(C) 1982-2012 IEEE. Calibration is an essential prerequisite for the combined application of light detection and ranging (LiDAR) and inertial measurement unit (IMU). However, current LiDAR-IMU calibration usually relies on particular artificial targets or facilities and the intensive labor greatly limits the calibration flexibility. For these reasons, this article presents a novel multifeature based on-site calibration method for LiDAR-IMU system without any artificial targets or specific facilities. This new on-site calibration combines the point/sphere, line/cylinder, and plane features from LiDAR scanned data to reduce the labor intensity. The main contribution is that a new method is developed for LiDAR extrinsic parameters on-site calibration and this method could incorporate two or more calibration models to generate more accurate calibration results. First of all, the calibration of LiDAR extrinsic parameters is performed through estimating the geometric features and solving the multifeature geometric constrained optimization problem. Then, the relationships between LiDAR and IMU intrinsic calibration parameters are determined by the coordinate transformation. Lastly, the full information maximum likelihood estimation (FIMLE) method is applied to solve the optimization of the IMU intrinsic parameters calibration. A series of experiments are conducted to evaluate the proposed method. The analysis results demonstrate that the proposed on-site calibration method can improve the performance of the LiDAR-IMU.
\end{abstract}

\section{Disciplines}

Engineering | Science and Technology Studies

\section{Publication Details}

Liu, W., Li, Z., Malekian, R., Sotelo, M., Ma, Z. \& Li, W. (2020). A Novel Multifeature Based On-Site Calibration Method for LiDAR-IMU System. IEEE Transactions on Industrial Electronics, 67 (11), 9851-9861.

\section{Authors}

Wanli Liu, Zhixiong Li, Reza Malekian, Miguel Sotelo, Zhenjun Ma, and Weihua Li 


\section{A Novel Multi-Feature based On-Site Calibration Method for LiDAR-IMU System}

\begin{abstract}
Calibration is an essential prerequisite for the combined application of Light Detection and Ranging (LiDAR) and Inertial Measurement Unit (IMU). However, current LiDARIMU calibration usually relies on particular artificial targets or facilities and the intensive labor greatly limits the calibration flexibility. For these reasons, this paper presents a novel multifeature based on-site calibration method for LiDAR-IMU system without any artificial targets or specific facilities. This new on-site calibration combines the point/sphere, line/cylinder and plane features from LiDAR scanned data to reduce the labor intensity. The main contribution is that a new method was developed for LiDAR extrinsic parameters on-site calibration and this method could incorporate two or more calibration models to generate more accurate calibration results. First of all, the calibration of LiDAR extrinsic parameters is performed through estimating the geometric features and solving the multi-feature geometric constrained optimization problem. Then, the relationships between LiDAR and IMU intrinsic calibration parameters are determined by the coordinate transformation. Lastly, the FIMLE method is applied to solving the optimization of the IMU intrinsic parameters calibration. A series of experiments have been conducted to evaluate the proposed method. The analysis results demonstrate that the proposed on-site calibration method can improve the performance of the LiDAR-IMU.
\end{abstract}

Index Terms-LiDAR, IMU, on-site calibration, multi-feature, full information maximum likelihood estimation

\section{I.INTRODUCTION}

$I^{\prime}$ the modern society, Light Detection and Ranging (LiDAR) and Inertial Measurement Unit (IMU) can be usually found on vehicles, personal electronic devices and robots; they are also increasingly used to perform locating and navigating task. However, due to the time drift, IMU is usually unreliable to estimate the position and the attitude over long periods of time, producing large accumulated errors. LiDAR is an optical metrological technology that captures target surfaces in the three-dimensional (3D) space with highly redundant sets of discrete points. It provides 3D position information with higher accuracy and efficiency, thereby reducing or bounding IMU drift. Therefore, the combination of LiDAR and IMU could improve the estimated robustness and the calibrated accuracy, also it is very suitable in many applications, especially in Global Position System (GPS) denied environments [1-4].

To guarantee the optimal performance, proper calibration is a precondition for LiDAR-IMU applications, and its measurement performance can be improved greatly after the calibration. Usually, the LiDAR-IMU calibration mainly includes the LiDAR extrinsic parameters calibration, IMU intrinsic parameters calibration, and coordinate transformation between LiDAR and IMU. However, to the best of the authors' knowledge, the current calibration technologies have rarely been investigated for LiDAR-IMU calibration. The possible reasons are that, the new development 3D LiDAR, such as the revolving-head LiDAR (Velodyne LiDAR series), consists of multi-layer laser rangefinders, which makes the transformation model between LiDAR and IMU is extremely complicated. In addition, the measurement information of LiDAR and IMU cannot be compared directly. For these reasons, the procedure to calibrate a LiDAR-IMU system directly has rarely been proposed in the literatures; instead, they mainly focus on the calibration of 3D LiDAR itself, or 2D LiDAR-IMU system. The features used during on-site calibration often rely on particular artificial targets or specific facilities [5-7]. For example, Marcelo et al. [8] and Chen et al. [9] used the moving artificial sphere targets to calibrate the multiple layer LiDAR and 2D LiDAR-IMU system. Kang et al. [10] , Gerardo et al. [11] and Huang et al. [12] used the artificial simple planes, pattern planes and V-shaped planes, to calibrate the 2D LiDAR, 2D LiDARIMU system and multiple layer LiDAR, respectively. Shang et al. [13] and Zhou et al. [14] used an orthogonal normal vector pair and three plane-line to calibrate the 3D LiDAR and 2D LiDAR-IMU system, respectively. Chan et al. [15] investigated the on-site calibration of the multi-layer LiDAR using the cylinder target in static and kinematic modes. Liu et al. [16] proposed a method using cone-cylinder targets for extrinsicintrinsic calibration of a LiDAR-IMU. Also the LiDAR manufacturers provide the specific calibration facilities, such as Leica white and black color planar targets and FARO different diameter sphere targets [17-18].

As mentioned above, although the particular artificial targets or additional facilities can be used to calibrate the LiDAR-IMU system on-site, they usually have the followings drawbacks [1923]: (1) the time and cost to set up and maintain a certain quantity of artificial targets field are very huge; (2) finding a sufficient free space on the job site to calibrate the LiDAR-IMU system is usually very difficult; (3) they do not have the ability to solve the on-site real-time calibration problem for LiDARIMU system; for example, in order to maintain the higher measurement accuracy for long periods of time in performing navigation or localization tasks, the LiDAR-IMU systems with measuring instability should be calibrated automatically and frequently.

These drawbacks significantly limit the on-site calibration flexibility for LiDAR-IMU system [24-25]. In order to overcome these drawbacks, the existing natural features can be utilized to perform the on-site calibrating of LiDAR-IMU, which is similar to the case of using artificial targets. Usually, the features of artificial targets, such as point/sphere, line/cylinder and plane features can be easily found in many indoor and outdoor natural scenes, and these features can be detected automatically based on a specification algorithm. 
However, when high accuracy calibration requirement needs to be met, the single feature in natural scenes can be difficult to achieve this requirement. This is because the single point-based calibration may suffer from the parameter correlation, the single plane-based calibration accuracy will be very poor due to the low diversity of the laser scanned data in horizontal orientation, and the single line-based calibration is subject to non-linear error [26-27].

For this reason, a multi-feature based method which combines the advantages of point/sphere, line/cylinder and plane features from LiDAR scanned data is proposed for on-site calibration of a LiDAR-IMU system without using any artificial targets or specific facility. Moreover, the line features can be derived from the intersection of a set of plane features to produce a line, and point features can be obtained from the intersection of line features, et.al. Also these features can be easily found in indoor and outdoor environments [28-29].

The main contributions of this research are summarized as follows: (1) a new on-site calibration method based on multiple features for LiDAR extrinsic parameters is developed. This calibration method can incorporate two or more calibration models (such as point/sphere, line/cylinder and plane features) to generate more accurate calibration results. Also it can be applied to calibrate any type of 3D or 2D LiDAR-IMU system. (2) The FIMLE method is present to solve the IMU intrinsic parameters optimization problem. The FIMLE is robust to deal with the uncertainty of the propagation intrinsic parameters vector of LiDAR-IMU.

The on-site calibration procedure for LiDAR-IMU is carried out through the following steps: First of all, the LiDAR extrinsic parameter is calibrated through registering the geometric features extracted from LiDAR scanned data and solving the multi-feature geometric constrained optimization problem. Secondly, the relationships between the extrinsic parameters and the intrinsic parameters of LiDAR-IMU are determined by the coordinate transformation. Finally, the FIMLE method is applied to resolve optimization problem of the IMU intrinsic parameter calibration. The simulation and experimental results show that extrinsic and intrinsic error parameters of LiDAR-IMU can be accurately calibrated and the overall performances are obviously improved after calibration using the multi-feature based method.

\section{II.RELATIONSHIP BETWEEN LIDAR AND IMU}

Generally, LiDAR-IMU system includes three basic coordinate frames: LiDAR frame $\{L\}$, IMU frame $\{I\}$, and Earth frame $\{E\}$. Figure 1 gives the relationships of the three frames. Supposing that a target point ${ }^{E} P$ in frame $\{E\}$ is corresponding to a point ${ }^{L} P$ in frame $\{L\}$, the position of ${ }^{E} P$ in frame $\{L\}$ can be computed as

$$
{ }^{L} P=R_{E}^{L E} P+T_{E}^{L}
$$

where $R_{E}^{L}$ and $T_{E}^{L}$ are the orthonormal rotation matrix and translation matrix between frame $\{E\}$ and frame $\{L\}$.

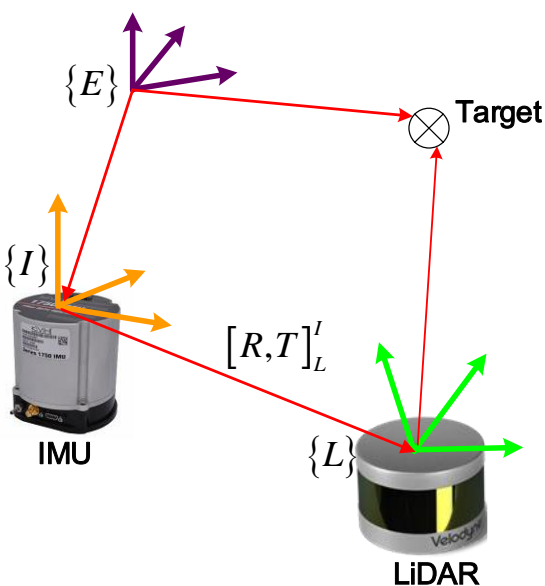

Fig. 1. Relationship between frames $\{L\},\{I\}$ and $\{E\}$.

Usually, IMU consists of three gyros and three accelerometers. The accelerometer outputs the specific force and the gyro provides the changing of Euler angles. Based on the information from the gyro and the accelerometer, the position of IMU can be obtained. The point ${ }^{I} P$ in frame $\{I\}$ converts to the point ${ }^{E} P$ in frame $\{E\}$ as:

$$
{ }^{E} P=R_{I}^{E I} P+T_{I}^{E}
$$

where $R_{I}^{E}$ and $T_{I}^{E}$ are the rotation matrix and translation matrix from frame $\{I\}$ to frame $\{E\}$

Substituting Equation (2) into Equation (1), and note that ${ }^{L} P$ in frame $\{I\}$ can be expressed as ${ }^{L} P=R_{I}^{L I} P+T_{I}^{L}$, we obtain

$$
{ }^{L} P=R_{E}^{L}\left(R_{I}^{E I} P+T_{I}^{E}\right)+T_{E}^{L}=R_{I}^{L I} P+T_{I}^{L}
$$

where $R_{I}^{L}$ and $T_{I}^{L}$ are the rotation matrix and translation matrix from frame $\{I\}$ to frame $\{L\}$

Equation (3) is the coordinate transforming relationship between LiDAR and IMU. This research focuses on the LiDAR-IMU system calibration, and the aim is to improve the accuracy of LiDAR extrinsic parameters, IMU intrinsic parameters, $R_{I}^{L}$ and $T_{I}^{L}$.

In on-site calibration, the LiDAR extrinsic parameters can be calibrated through observing the true values of ${ }^{E} P$ provided through higher accuracy instruments in frame $\{E\}$. However, the LiDAR scanning is the sparse sampled of 3D points cloud on the target's surfaces, and hard to realize the direct point-topoint matching. But the intrinsic geometric feature of LiDAR scanned 3D points date can be more reliable in obtaining the correspondence in different data sets. In the next sections, the different type of constrained geometric feature will be given and used to calibrate the LiDAR-IMU system on-site.

\section{III.MULTI-TYPE GEOMETRIC FEATURES}

Supposing that the LiDAR 3D point dataset $P=\left\{x_{i}, y_{i}, z_{i}\right\}$ has a certain geometric shape which may be a point/sphere, a line/cylinder or a plane, the functional model of $P$ can be expressed as [30]:

$$
F^{\Phi}(P)=0
$$

where $\Phi$ is the type of the geometric shapes, $F^{\Phi}$ is the geometric function of the $\Phi$, and $F^{\Phi}=0$ represents the geometric features which could be found by fitting on the 3D points. 


\section{A. Point/Sphere Features}

The points and spheres can be treated as one category because a point is defined as a sphere with a radius of zero. Supposing $\left(x_{c}, y_{c}, z_{c}\right)$ is the center coordinates of the sphere, and $R$ is the radius of the sphere with a specified "fixed" radius. The sphere equation is denoted as:

$$
\left(x_{i}-x_{c}\right)^{2}+\left(y_{i}-y_{c}\right)^{2}+\left(z_{i}-z_{c}\right)^{2}-R^{2}=0
$$

In consideration of the on-site calibration, the spherical feature is taken as an optimization problem, which is to minimize the distance from point dataset $P$ to the fitted sphere. Thus, the constrained function can be expressed as:

$$
\sum_{i}\left(\sqrt{\left(x_{i}-x_{c}\right)^{2}+\left(y_{i}-y_{c}\right)^{2}+\left(z_{i}-z_{c}\right)^{2}}-R\right)^{2}=\sum_{i} v_{i}^{2}=\min
$$

The residuals $v_{i}$ can be linearized as:

$$
v_{i}=\frac{x_{i}-x_{c}}{d_{i}} d x_{c}+\frac{y_{i}-y_{c}}{d_{i}} d y_{c}+\frac{z_{i}-z_{c}}{d_{i}} d z_{c}+R_{i}^{0}-R
$$

where

$$
d_{i}=\sqrt{\left(x_{i}-x_{c}\right)^{2}+\left(y_{i}-y_{c}\right)^{2}+\left(z_{i}-z_{c}\right)^{2}}
$$

$R_{i}^{0}=\sqrt{\left(x_{i}-x_{c}^{2}\right)^{2}+\left(y_{i}-y_{c}^{2}\right)^{2}+\left(z_{i}-z_{c}^{2}\right)^{2}}$.

According to Equation (7), if the number of points $P$ is $n$, the total error constrained function of spherical feature can be denoted as:

$$
\text { where } W=\left(\begin{array}{ccc}
\underset{n \times 1}{V}=\underset{n \times 3 \times 1}{W} \underset{n \times 1}{d_{1}} & \frac{y_{1}-y_{c}}{d_{1}} & \frac{z_{1}-z_{c}}{d_{1}} \\
\mathrm{M} & \mathrm{M} & \mathrm{M} \\
\frac{x_{n}-x_{c}}{d_{n}} & \frac{y_{n}-y_{c}}{d_{n}} & \frac{z_{n}-z_{c}}{d_{n}}
\end{array}\right), X=\left(\begin{array}{c}
d x_{c} \\
d y_{c} \\
d z_{c}
\end{array}\right), L=\left(\begin{array}{c}
R-R_{1}^{0} \\
\mathrm{M} \\
R-R_{n}^{0}
\end{array}\right)
$$

\section{B. Line/Cylinder Features}

Typically, the line and cylinder can be taken as one category because the line can be considered as a cylinder with a radius of zero. Suppose the model of 3D line is calculated using six descriptive parameters

$$
\left\{\begin{array}{l}
x=x_{0}+\lambda u_{x} \\
y=y_{0}+\lambda u_{y} \\
z=z_{0}+\lambda u_{z}
\end{array}\right.
$$

where $\lambda$ denotes a scale factor, $\left(u_{x}, u_{y}, u_{z}\right)$ represent the direction vector components of $3 \mathrm{D}$ line, and $\left(x_{0}, y_{0}, z_{0}\right)$ denote the point coordinates along the $3 \mathrm{D}$ line.

Otherwise, a cylinder can be described using seven parameters; that is to say, one parameter denotes the cylinder radius $r$ with $\sqrt{d_{x}^{2}+d_{y}^{2}+d_{z}^{2}}-r=0, d_{i}=\sqrt{d_{x}^{2}+d_{y}^{2}+d_{z}^{2}}$ is the components of the distance from the point in question to the cylinder axis, other six parameters denote the cylinder axis.

Towards a series of $n$ points, $n \geq 2$ represents a line and $n \geq 5$ (non-planar/non-collinear points) represents a cylinder. Therefore, the constrained function of the line/cylinder can be represented as:

$$
\sum_{i=1}^{n}\left(\sqrt{\lambda_{i}^{2} d_{i}^{2}-r_{i}^{2}}\right)=\min \left(u_{x}, u_{y}, u_{z}, x_{0}, y_{0}, z_{0}, r\right)
$$

\section{Plane Features}

Generally, the function of plane feature is represented as:

$$
A x+B y+C z+D=0
$$

where $D$ denotes the perpendicular distance between the plane and the origin of 3D coordinate system; $A, B$ and $C$ represent the unit vector components of a normal plane.

In order to estimate the parameters of the best-fit plane, the least square method is used to illustrate the plane fitting as:

$$
d_{i}=\frac{A x_{i}+B y_{i}+C z_{i}+D}{\sqrt{A^{2}+B^{2}+C^{2}}}
$$

Towards a series of $n$ points, when the number of noncollinear points $n \geq 3$, the constrained function of the plane can be calculated as:

$$
\sum_{i=1}^{n} d_{i}^{2}=\min (A, B, C, D)
$$

\section{Multi-Feature Constrained Confidence Matching}

The multi-feature constrained confidence matching is important to evaluate the matching reliability of the geometric shape, and the matching interval can also be used to control the deviation of geometric features.

Suppose the confidence matching interval is $(\mu, \sigma)$, which can be applied to evaluate the matching properties and noise errors of the LiDAR scanned 3D points dataset. To ensure that the geometric function $F^{\Phi}(P)$ could be equal to zero, the error covariance model of $(\mu, \sigma)$ is defined as:

$$
\left\{\begin{array}{l}
\mu=E\left(F^{\Phi}(P)^{2}\right) \\
\sigma=D\left(F^{\Phi}(P)^{2}\right)
\end{array}\right.
$$

where $E$ and $D$ represent the expectation and deviation of $F^{\Phi}(P)^{2}$.

According to Equation (4) and Equation (14), if the confidence matching error exists, it will yield deviations in geometric shapes. Therefore, the estimation of the geometric features could be affected by the confidence matching error. However, the type of the above defined geometric features will keep consistent in case of the confidence matching. It can assume that the geometric features are constraints in a short range and then the corresponding 3D point of the features will fulfill the invertible affine transformation. Although a minor deviation may appear in the calibration, its influence can be reduced by the error covariance model of $(\mu, \sigma)$.

\section{IV.CALIBRATION PARAMETERS}

\section{A. LIDAR Extrinsic Calibration Parameters}

Currently, different types of LiDAR exist with varying scanning mechanisms, number of lasers, and geometric configurations. In this paper, a Velodyne VLP-16 is used, as shown in Figure 2. The VLP-16 LiDAR possesses high accuracy and definition, it can provides a $360^{\circ}$ horizontal fields of view $(\theta)$ and $30^{\circ}$ vertical fields of view $(\alpha)$, also a rich point cloud be captured at a sampling rate of three million points per second [31]. 


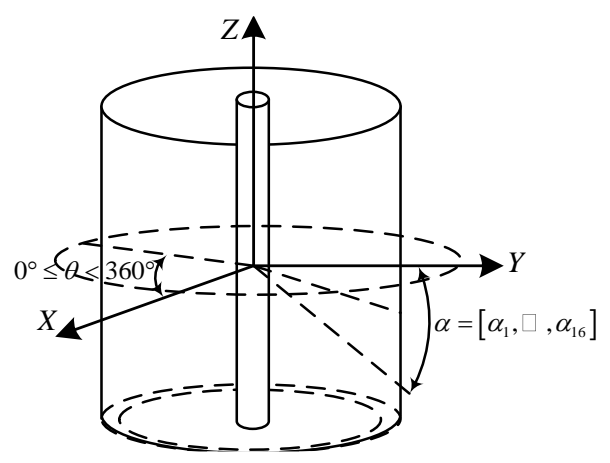

Fig. 2. VLP-16 LiDAR geometric configuration.

The basic model of VLP-16 LiDAR for the point data generation process is defined in a spherical global coordinate system. The mathematical model for measuring 3D point data can be modeled as follows:

$$
L_{i}(\rho, \alpha, \theta)=\left(\begin{array}{c}
x_{i} \\
y_{i} \\
z_{i}
\end{array}\right)=\left(\begin{array}{c}
\rho_{i} \cos \alpha_{i} \sin \theta_{i} \\
\rho_{i} \cos \alpha_{i} \cos \theta_{i} \\
\rho_{i} \sin \alpha_{i}
\end{array}\right)
$$

where $\alpha$ denotes the LiDAR fixed vertical fields of view angle, $\theta$ is the horizontal fields of view angle, and $\rho$ represents the measuring range.

According to Equation (15), the mathematical model for 3D point position on the unit sphere would be converted into Cartesian coordinates; however this conversion will cause the additional error for LiDAR extrinsic parameters, the error model is expressed as:

$$
\bar{L}_{i}(\rho, \alpha, \theta)=\left(\begin{array}{c}
\left(k_{L} \rho_{i}-\Delta \rho_{i}\right) \cos \alpha_{i} \sin \theta_{i}-\Delta h_{i} \cos \left(\theta_{i}-\Delta \theta_{i}\right) \\
\left(k_{L} \rho_{i}-\Delta \rho_{i}\right) \cos \alpha_{i} \cos \theta_{i}+\Delta h_{i} \sin \left(\theta_{i}-\Delta \theta_{i}\right) \\
\left(k_{L} \rho_{i}-\Delta \rho_{i}\right) \sin \alpha_{i}+\Delta v_{i}
\end{array}\right) \cdot\left(\begin{array}{ccc}
\cos \theta_{i} & -\sin \theta_{i} & 0 \\
\sin \theta_{i} & \cos \theta_{i} & 0 \\
0 & 0 & 1
\end{array}\right)
$$

where $\bar{L}$ is the actual LiDAR value; $\Delta \rho$ denotes the offset correction of the LiDAR measurement range; $\Delta \theta$ represents offset correction of view angle in horizontal fields ; $\Delta h$ denotes horizontal offset correction; $\Delta v$ represents vertical offset correction; and $k_{L}$ denotes the scales factor; $i=1, \ldots, 16$ is the number of laser rangefinder.

Suppose $R_{\theta_{i}}$ represents the rotation matrix of $\theta$, the Equation (16) is rewritten as [30]

$$
\bar{L}_{i}(\rho, \alpha, \theta)=R_{\theta_{i}}\left[k_{L} \rho_{i}\left(\begin{array}{c}
\cos \alpha_{i} \sin \theta_{i} \\
\cos \alpha_{i} \cos \theta_{i} \\
\sin \alpha_{i}
\end{array}\right)-\left(\begin{array}{c}
\Delta \rho_{i} \cos \alpha_{i} \sin \theta_{i}+\Delta x_{i} \cos \left(\theta_{i}-\Delta \theta_{i}\right) \\
\Delta \rho_{i} \cos \alpha_{i} \cos \theta_{i}-\Delta x_{i} \sin \left(\theta_{i}-\Delta \theta_{i}\right) \\
\Delta \rho_{i} \sin \alpha_{i}-\Delta z_{i}
\end{array}\right)\right]
$$

From Equation (15) to Equation (17), the LiDAR extrinsic calibration parameters vector $\Theta_{L}$ was given as follows:

$$
\Theta_{L}=[\rho, \alpha, \theta, k]^{T}
$$

\section{B. IMU Intrinsic Calibration Parameters}

According to the references [32-33], the measurement error functions of IMU can be expressed as:

$$
\left[\begin{array}{l}
\bar{I}_{a_{x}} \\
\bar{I}_{a_{y}} \\
\bar{I}_{a_{z}}
\end{array}\right]=\left[\begin{array}{ccc}
1+k_{\alpha, x}+\delta k_{\alpha, x} & \delta_{\alpha, x y} & \delta_{\alpha, x z} \\
\delta_{\alpha, y x} & 1+k_{\alpha, y}+\delta k_{\alpha, y} & \delta_{\alpha, y z} \\
\delta_{\alpha, z x} & \delta_{\alpha, z y} & 1+k_{\alpha, z}+\delta k_{\alpha, z}
\end{array}\right]\left[\begin{array}{c}
I_{a_{x}} \\
I_{a_{y}} \\
I_{a_{z}}
\end{array}\right]+\left[\begin{array}{c}
b_{a_{x}}+\delta b_{a_{x}} \\
b_{a_{y}}+\delta b_{a_{y}} \\
b_{a_{z}}+\delta b_{a_{z}}
\end{array}\right]+\left[\begin{array}{c}
w_{a_{x}} \\
w_{a_{y}} \\
w_{a_{z}}
\end{array}\right]
$$

$$
\begin{aligned}
{\left[\begin{array}{l}
\bar{I}_{g_{x}} \\
\bar{I}_{g_{y}} \\
\bar{I}_{g_{z}}
\end{array}\right]=\left[\begin{array}{ccc}
1+k_{g, x}+\delta k_{g, x} & \delta_{g, x y} & \delta_{g, x z} \\
\delta_{g, y x} & 1+k_{g, y}+\delta k_{g, y} & \delta_{g, y z} \\
\delta_{g, z x} & \delta_{g, z y} & 1+k_{g, z}+\delta k_{g, z}
\end{array}\right]\left[\begin{array}{l}
I_{g_{x}} \\
I_{g_{y}} \\
I_{g_{z}}
\end{array}\right] } \\
+\left[\begin{array}{c}
b_{g_{x}}+\delta b_{g_{x}} \\
b_{g_{y}}+\delta b_{g_{y}} \\
b_{g_{z}}+\delta b_{g_{z}}
\end{array}\right]+\left[\begin{array}{l}
w_{g_{x}} \\
w_{g_{y}} \\
w_{g_{z}}
\end{array}\right]+\left[\begin{array}{ccc}
\xi b_{g_{x}} & 0 & 0 \\
0 & \xi b_{g_{y}} & 0 \\
0 & 0 & \xi b_{g_{z}}
\end{array}\right]\left[\begin{array}{l}
I_{a_{x}} \\
I_{a_{y}} \\
I_{a_{z}}
\end{array}\right]
\end{aligned}
$$

where $\xi b_{g}$ represents the coefficient of g-dep bias; $b_{a}$ and $b_{g}$ denote the biases of accelerometer and gyroscope, respectively; $\delta_{a}$ and $k_{a}$ represent accelerometer misalignment biased and scale factors; $\delta_{g}$ and $k_{g}$ denote gyroscope misalignment biased and scale factors; $I_{a}$ and $I_{g}$ denote the actual accelerometer and gyroscope values, respectively; $\bar{I}_{a}$ and $\bar{I}_{g}$ denote accelerometer and gyroscope outputs; $w_{a}$ and $w_{g}$ denote accelerometer and gyroscope noises; $\delta k_{\alpha}$ and $\delta k_{g}$ denote accelerometer and gyroscope scale factors instability, respectively.

According to Equation (19) to Equation (20), the IMU intrinsic calibration parameters vectors $\Theta_{a}$ and $\Theta_{g}$ are given as:

$$
\begin{gathered}
\Theta_{a}=\left[k_{a_{x}}, k_{a_{y}}, k_{a_{z}}, b_{a_{x}}, b_{a_{y}}, b_{a_{z}}, b_{a_{x}}, w_{a_{x}}, w_{a_{y}}, w_{a_{z}}\right]^{T} \\
\Theta_{g}=\left[k_{g_{x}}, k_{g_{y}}, k_{g_{z}}, b_{g_{x}}, b_{g_{y}}, b_{g_{z}}, b_{g_{x}}, w_{g_{x}}, w_{g_{y}}, w_{g_{z}}\right]^{T}
\end{gathered}
$$

\section{V.ON-SITE CALIBRATION FOR LIDAR-IMU}

The on-site calibration process is shown in Figure 3. It mainly includes calibration of LiDAR extrinsic parameters and IMU intrinsic parameters, and optimization of LiDAR-IMU system parameters. The detailed calibration procedure is implemented as follows:

\section{A. Calibration of LiDAR Extrinsic Parameters}

As defined in section III, a geometric feature can be represented as:

$$
G=\left\{P, \Phi, F^{\Phi},(\mu, \sigma)\right\}
$$

where $\Phi \in\{$ point/ sphere, line / cylinder, plane $\}$ is the type of the geometric shapes, $P$ is the $3 \mathrm{D}$ point dataset, $F^{\Phi}$ is the geometric function of the $\Phi$, and $(\mu, \sigma)$ is the confidence matching interval.

In the process of geometric feature extraction from the LiDAR scanned point datasets, for a specific type of geometric shape $\Phi$, the geometric feature $G_{\Phi}$ can be estimated through calculating a geometric function of $F^{\Phi}$ on the LiDAR scanned $3 \mathrm{D}$ point dataset using the developed geometric features, and the confidence matching interval $(\mu, \sigma)$ can be estimated similarly. Thus, a set of geometric features $G_{\Phi}$ can be obtained and used for the calibration of LiDAR extrinsic parameters.

During the calibration of LiDAR extrinsic parameters, the LiDAR estimated calibration parameters $\Theta_{L}$ are always used to generate the $3 \mathrm{D}$ points cloud data, and to extract the geometric feature from the scanned point dataset. The extracted 
geometric shape will contain the calibration parameters error, which can be deviated by solving the multi-feature geometric constrained optimization problem.

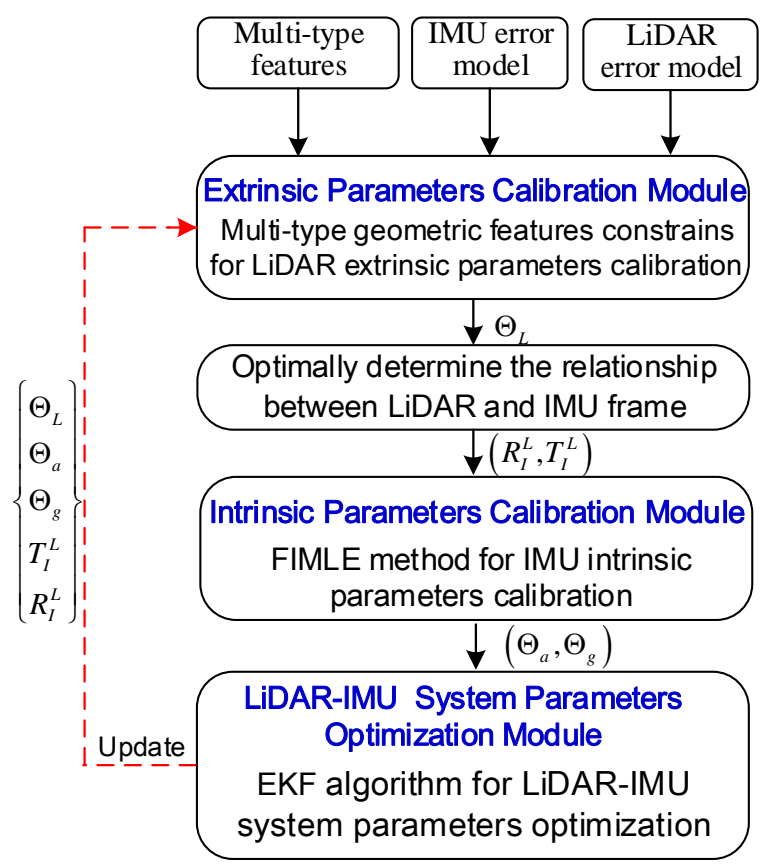

Fig. 3. On-site calibration procedure for LiDAR-IMU system.

The calibration procedures are implemented as follows. Generally, an important step in LiDAR measurement is to estimate the error of the calibration parameters from LiDAR scanned points dataset. Firstly, the 3D points data from LiDAR scanning can be applied to determine the geometric shape. When the LiDAR scanned points mainly lie on the $i^{\text {th }}$ target surface, the scanned geometric feature parameters can be computed through minimizing the linear least square problems as follows:

$$
\Theta_{L}=\arg \min \sum_{i=1}^{n}\left\|\left.G_{\Phi}^{i}\right|_{\text {True }}-\left.G_{\Phi}^{i}\right|_{\text {Estimate }}\right\|^{2}
$$

where $\left.G_{\Phi}^{i}\right|_{\text {True }}$ and $\left.G_{\Phi}^{i}\right|_{\text {Estimate }}$ are the nominal and estimated values of a certain geometric feature.

When the multi-type geometric shapes are determined in relation to the LIDAR frame, the optimal calibration parameters error $\hat{\Theta}_{L}$ can be computed as:

$$
\hat{\Theta}_{L}=\left(\begin{array}{l}
\left\{\arg \min \sum_{i=1}^{n}\left\|\left.G_{\Phi_{\text {Point }}}^{i}\right|_{\text {True }}-\left.G_{\Phi_{\text {Point }}}^{i}\right|_{\text {Estimate }}\right\|^{2}\right\} \otimes \\
\left\{\arg \min \sum_{i=1}^{n}\left\|\left.G_{\Phi_{\text {Line }}}^{i}\right|_{\text {True }}-\left.G_{\Phi_{\Phi_{\text {Line }}}}^{i}\right|_{\text {Estimate }}\right\|^{2}\right\} \\
\left\{\arg \min \sum_{i=1}^{n}\left\|\left.G_{\Phi_{\text {Plane }}}^{i}\right|_{\text {True }}-\left.G_{\Phi_{\text {Plane }}}^{i}\right|_{\text {Estimate }}\right\|^{2}\right\}
\end{array}\right)
$$

According to the LiDAR scanned multi-type geometric features, Equation (25) is able to compute as a nonlinear optimization problem, which uses the previously calculated results as initial values to optimally estimate the LiDAR extrinsic calibration parameters.

\section{B. IMU Intrinsic Parameters Calibration}

Next, the FIMLE method will be used to calibrate IMU intrinsic parameter. The FIMLE method is based on the maximum likelihood estimation principle and can combine with the transformation matrix vector to deal with the uncertainty and incompleteness for IMU intrinsic parameters calibration. Also it can provide the accurate estimation for IMU measurement error equations and improve the calibration accuracy and reliability.

According to Equation (2), Equation (21) and Equation (22), the IMU intrinsic parameters vector and the coordinate transformation matrix vector can be expressed as:

$$
\left\{\begin{array}{l}
\Omega=\left[\begin{array}{ll}
\Theta_{\alpha} & \Theta_{g}
\end{array}\right]^{T} \\
M=\left[\begin{array}{ll}
R_{I}^{L} & T_{I}^{L}
\end{array}\right]^{T}
\end{array}\right.
$$

where the $\Omega$ denotes IMU intrinsic parameters vector, $M$ represents the coordinate transformation matrix vector between LiDAR and IMU; $T_{I}^{L}, R_{I}^{L}$ represent the translating and rotating matrix between IMU and LiDAR frames.

The architecture of FIMLE method is shown in Figure 3. The main purpose of using FIMLE to calibrate IMU intrinsic parameter is to solve the following optimization equations:

$$
\begin{gathered}
M_{F I M L E}, \Omega_{F I M L E}=\underset{M, \Omega}{\arg \min } f(M, \Omega, U, V) \\
f(M, \Omega, U, V)=\prod_{k=0}^{K} f\left(U_{k} \mid M_{k}, \Omega\right) \times \prod_{k=0}^{K} f\left(V_{k} \mid M_{k}, \Omega\right)
\end{gathered}
$$

where $f(\cdot)$ represents the joint probability density function; $M_{\text {FIMLE }}$ denotes the FIMLE value of the coordinate transformation matrix vector $M ; \Omega_{F I M L E}$ represents the FIMLE value of IMU intrinsic parameters vector $\Omega ; U$ denotes the optimum values for coordinate transformation matrix vector $M ; V$ represents the measurement optimum values for IMU intrinsic parameters vector $\Omega ; k \in[0, K]$ is the calibration time instances.

The calibration procedure of the IMU intrinsic parameter calibration using FIMLE method is implemented as:

(1) Estimation nominal values of $M$ and $\Omega$

According to Equation (19) and Equation (20), the estimation nominal values of $M$ and $\Omega$ can be calculated as follows:

$$
\left\{\begin{array}{l}
\bar{M}=M \otimes \delta M \\
\bar{\Omega}=\Omega \otimes \delta \Omega
\end{array}\right.
$$

where $\bar{M}$ and $\bar{\Omega}$ are the nominal estimation values of $M$ and $\Omega$, respectively; $\delta M$ and $\delta \Omega$ are the error values of $M$ and $\Omega$; $\otimes$ represents the simply component wise addition.

(2) Representation minimal states of $M$ and $\Omega$

According to the Equation (29), the minimal states function can be computed as:

$$
\left\{\begin{array}{l}
M=M+J(\bar{M}) w_{I M U} \\
\boldsymbol{\Omega}=H(\bar{M}) \cdot \Omega+J(\bar{\Omega}) w_{I M U}
\end{array}\right.
$$


where $M$ and $\$ 2$ represent the minimal states function of $M$ and $\Omega ; J$ denotes white noise processes vector regard to the minimal state $\mathbb{M} ; H$ denotes the derivative of $\Omega$ regard to $\Phi$ $; w_{I M U}=\left[\begin{array}{ll}w_{a} & w_{g}\end{array}\right]^{T}$ is the IMU noise processes vector.

(3) $M$ and $\Omega$ calibration

Based on Equation (27) to Equation (30), the calibration of $M$ and $\Omega$ using the FIMLE method can be equivalent to resolving the following functions optimization:

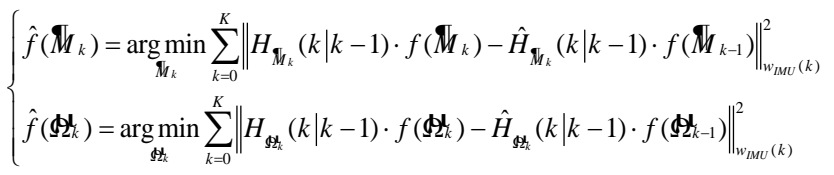

where \|\|$^{2}$ represents the vector Euclidean length; $\hat{f}(\cdot)$ denotes the minimal joint probability density function.

According to Equation (31), the optimal estimation of $M$ and $\Omega$ are obtained.

\section{LiDAR-IMU System Parameters Optimization}

Supposing $\Upsilon$ denotes LiDAR-IMU system parameters vector, it is expressed as follows:

$$
\Upsilon=\left[\begin{array}{lllll}
R_{I}^{L} & T_{I}^{L} & \Theta_{L} & \Theta_{\alpha} & \Theta_{g}
\end{array}\right]^{T}
$$

Based on the Extended Kalman Filter (EKF), the optimization parameters functions of LiDAR-IMU system are expressed as:

$$
\left\{\begin{array}{l}
R_{I}^{L}(k)=R_{I}^{L}(k-1)+\eta \cdot t \cdot(k-1) \\
T_{I}^{L}(k)=T_{I}^{L}(k-1)+t \cdot \eta(k-1) \\
\Theta_{L}(k)=\Theta_{L}(k-1)+\sigma_{\Theta_{L}} \cdot t \cdot(k-1) \\
\Theta_{\alpha}(k)=\Theta_{\alpha}(k-1)+\sigma_{\Theta_{\alpha}} \cdot t \cdot(k-1) \\
\Theta_{g}(k)=\Theta_{g}(k-1)+\sigma_{\Theta_{g}} \cdot t \cdot(k-1)
\end{array}\right.
$$

where $t$ denotes the calibrating time, $\sigma_{\Theta_{L}}, \sigma_{\Theta_{\alpha}}$ and $\sigma_{\Theta_{s}}$ represent the LiDAR and IMU (accelerometer, gyroscope) biases coefficient, respectively.

\section{VI.CALIBRATION USING SIMULATION DATASET}

In order to verify the effectiveness of the proposed method, three kinds of geometric features (point, line and plane) were simulated to evaluate the performance of the multi-feature based on-site calibration method. The simulation datasets were generated through estimating the point coordinate true values from intercepting the LiDAR-IMU measurement functions and geometric feature models. Geometric features can be derived from the surrounding environments in Figure 4, for example, the point features can be extracted from the spherical targets on the street lamp, the line features can be extracted from the street lamp poles or the intersection of two planes features, and the road or building wall surfaces can be extracted as the plane features.

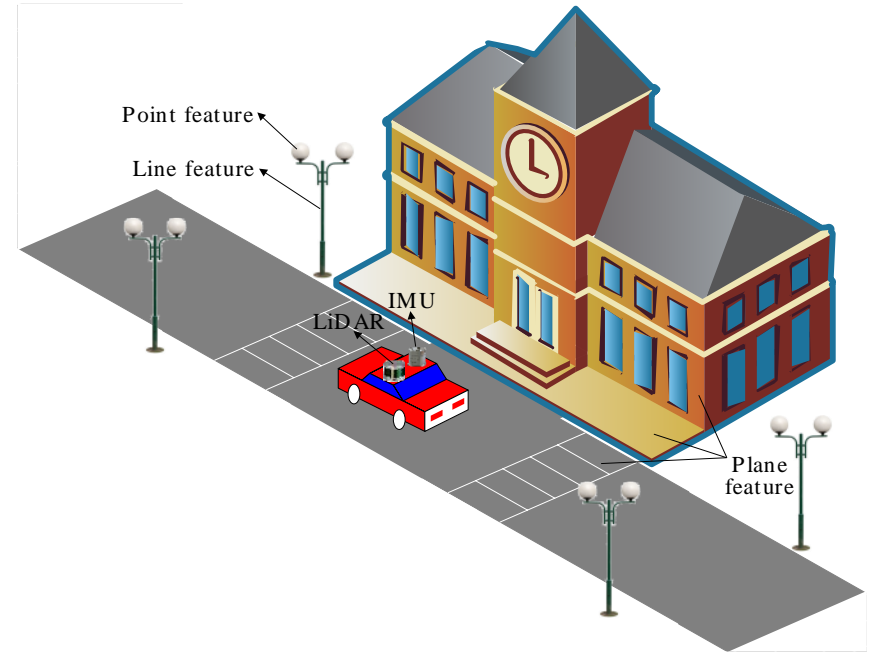

Fig. 4. Multi-feature calibration using simulation dataset

In the simulation, it is supposed that the reliability of the confidence matching interval of the multi-type geometric shape was very high, and no random noise was added. The calibration results by matching the multi-type geometric features using our proposed algorithm were shown in TABLE I and TABLE II.

In LiDAR extrinsic parameters simulated calibration experiments, the standard deviation value was set as $\rho=3.0$ $\mathrm{mm}, \alpha=\theta=0.03^{\circ}$. After calibration, the plane feature, line feature, point feature and multi-feature based method can improve the overall accuracy of $\rho$ by $34.67 \%, 37.33 \%, 45 \%$ and $61.33 \%$, respectively. Similarly, the overall accuracy improvements of $\alpha$ and $\theta$ are $23.33 \%, 33.33 \%, 36.67 \%$, $56.67 \%$, and $30 \%, 36.67 \%, 40 \%, 60 \%$,respectively.

TABLE I

THE VALUES OF LIDAR EXTRINSIC PARAMETERS AFTER SIMULATED CALIBRATION

\begin{tabular}{cccccc}
$\begin{array}{c}\text { LiDAR } \\
\text { extrinsic } \\
\text { parameters }\end{array}$ & $\begin{array}{c}\text { Before } \\
\text { calibration }\end{array}$ & $\begin{array}{c}\text { Plane } \\
\text { based } \\
\text { method }\end{array}$ & $\begin{array}{c}\text { Line } \\
\text { based } \\
\text { method }\end{array}$ & $\begin{array}{c}\text { Point } \\
\text { based } \\
\text { method }\end{array}$ & $\begin{array}{c}\text { Multi-feature } \\
\text { based method }\end{array}$ \\
$\rho(\mathrm{mm})$ & 3.0 & 1.96 & 1.88 & 1.65 & 1.16 \\
$\alpha(\mathrm{deg})$ & 0.03 & 0.023 & 0.02 & 0.019 & 0.013 \\
$\theta(\mathrm{deg})$ & 0.03 & 0.021 & 0.019 & 0.018 & 0.012 \\
\hline
\end{tabular}

In IMU intrinsic parameters simulated calibration experiments, the standard error value was set as $\delta_{a}=1.0 \mathrm{~m} / \mathrm{s}^{2}$, $\delta_{g}=1.0 \mathrm{rad} / \mathrm{s}$, and $k_{a}=k_{g}=0.1$. After calibration, the plane feature, line feature, point feature and multi-feature based method can improve the overall accuracy of $\delta_{a}$ by $14.9 \%$, $21.2 \%, 23.5 \%$ and $41.2 \%$, respectively. Similarly, the overall accuracy improvements of $\delta_{g}, k_{a}$ and $k_{g}$ are $32 \%, 37 \%, 39 \%$, $58 \%$, and $33.7 \%, 40.2 \%, 41.3 \%, 59.3 \%$, and $47 \%, 49 \%, 50 \%$, $61 \%$, respectively.

TABLE II

THE VALUES OF IMU INTRINSIC PARAMETERS AFTER SIMULATED CALIBRATION 


\begin{tabular}{cccccc}
\hline $\begin{array}{c}\text { IMU } \\
\text { intrinsic } \\
\text { parameters }\end{array}$ & $\begin{array}{c}\text { Before } \\
\text { calibration }\end{array}$ & $\begin{array}{c}\text { Plane } \\
\text { based } \\
\text { method }\end{array}$ & $\begin{array}{c}\text { Line } \\
\text { based } \\
\text { method }\end{array}$ & $\begin{array}{c}\text { Point } \\
\text { based } \\
\text { method }\end{array}$ & $\begin{array}{c}\text { Multi-feature } \\
\text { based method }\end{array}$ \\
\hline$\delta_{\alpha}\left(\mathrm{m} / \mathrm{s}^{2}\right)$ & 1.0 & 0.851 & 0.788 & 0.765 & 0.588 \\
$k_{\alpha}(\%)$ & 0.1 & 0.068 & 0.063 & 0.061 & 0.042 \\
$\delta_{g}(\mathrm{rad} / \mathrm{s})$ & 1.0 & 0.663 & 0.598 & 0.587 & 0.407 \\
$k_{g}(\%)$ & 0.1 & 0.053 & 0.051 & 0.05 & 0.039 \\
\hline
\end{tabular}

According to the results of simulated calibration experiments, it can be seen that the overall performances of LiDAR-IMU extrinsic and intrinsic parameters are obviously improved after applying the multi-feature based method.

\section{VII.EXPERIMENTAL VERIFICATION}

To validate the presented method using real datasets, a series of on-site calibration experiments were conducted. Figure 4 gives the experimental layouts, and the specifications of IMU and LiDAR are given in TABLE III. The calibration experiments were conducted with standard targets (sphere, cylinder and plane), LiDAR and IMU. In the experiments, the LiDAR and IMU were fixed on the rotated platform.

TABLE III

THE SPECIFICATIONS OF IMU AND LIDAR

\begin{tabular}{cccc}
\hline \hline Navigation Features & IMU & Performance Features & LiDAR \\
\hline $\begin{array}{c}\text { Horizontal Position } \\
\text { Accuracy }\end{array}$ & $0.5 \mathrm{~m}$ & Channels & 16 \\
$\begin{array}{c}\text { Vertical Position } \\
\text { Accuracy }\end{array}$ & $0.8 \mathrm{~m}$ & Range & $100 \mathrm{~m}$ \\
$\begin{array}{c}\text { Velocity Accuracy } \\
\text { Roll \& Pitch }\end{array}$ & $0.007 \mathrm{~m} / \mathrm{s}$ & Accuracy & $\pm 3 \mathrm{~cm}$ \\
Accuracy & $0.01^{\circ}$ & Vertical FOV & $30^{\circ}$ \\
$\begin{array}{c}\text { Heading Accuracy } \\
\text { Output Data Rate }\end{array}$ & $0.05^{\circ}$ & $\begin{array}{c}\text { Horizontal } \\
\text { FOV to }\end{array}$ & $360^{\circ}$ \\
$1000 \mathrm{~Hz}$ & $\begin{array}{c}\text { Output Data } \\
\text { Rate }\end{array}$ & $300,000 \mathrm{pts} / \mathrm{sec}$ \\
\hline \hline
\end{tabular}

\section{A. LIDAR Extrinsic Parameters Calibration Experiments}

In this experiment, in order to evaluate the effectiveness of the proposed method, the calibration performances of calibration methods based on point, line, plane and multifeature (including point, line and plane) were compared. As shown in Figure 4, LiDAR-IMU was used to capture 3D point datasets of the spherical features, cylinder features and planar features in all experiments. The high accurate total station instrument was used to determine the coordinate of each target in the frame $\{E\}$ from different observing orientations to obtain both high redundancy and accuracy.

In this test, the sphere target, cylinder target and plane target were located at $3 \mathrm{~m}$ away from the LiDAR-IMU system. After the LiDAR scanned 3D point datasets fully match the target geometric features, the LiDAR extrinsic parameters calibration can be solved as a nonlinear least squares problem using the

multi-type geometric feature constraints obtained from the geometric function $F^{\Phi}(P)$. The optimal estimation of LiDAR extrinsic parameters can be calculated according to Equation. (24) and (25).

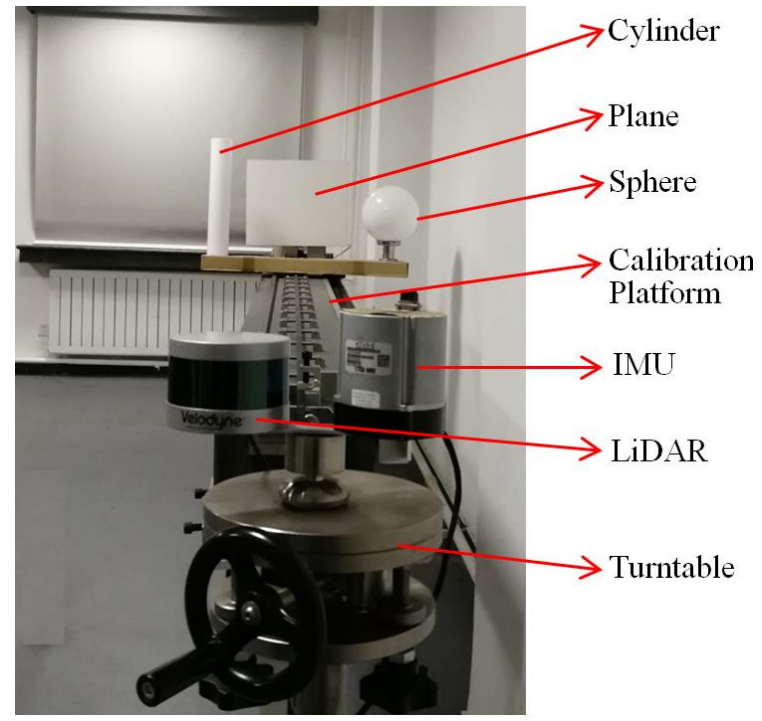

Fig. 4. The calibration experiment layouts

According to the scanned 3D point datasets of the sphere target, cylinder target, plane target and multi-type target, the geometric features could be extracted and compared with the true values given by the total station instrument. The extracted geometric features contain the LiDAR extrinsic parameters calibration error.

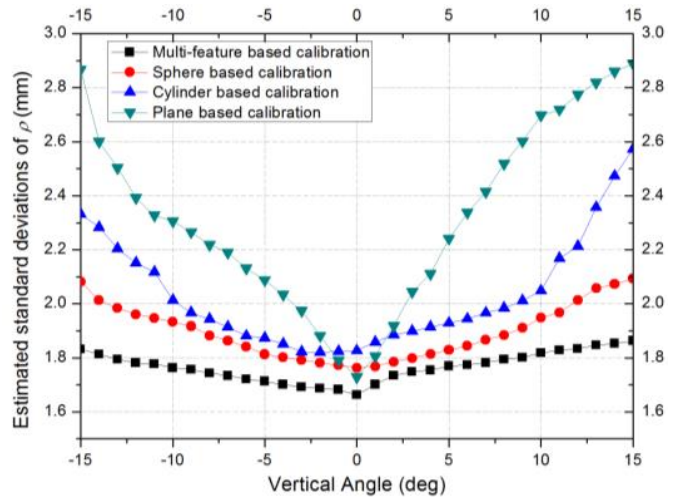

(a) The estimated standard deviation of $\rho$ VS vertical angle $\alpha$

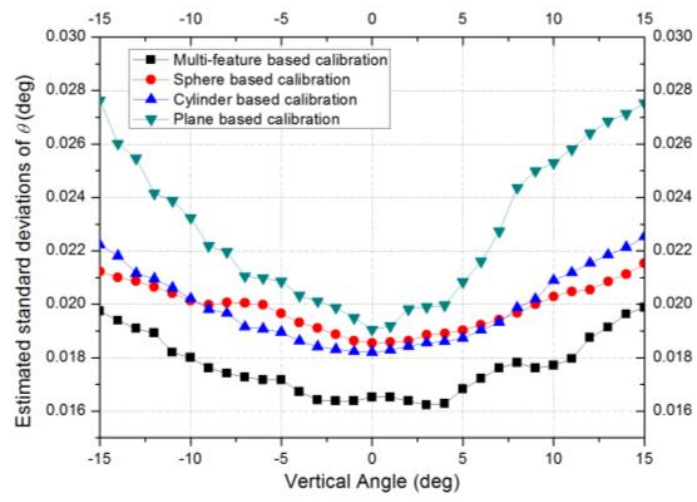

(b) The estimated standard deviation of $\theta$ VS vertical angle $\alpha$ Fig. 5. The calibration results of LiDAR extrinsic parameters 
Figure 5 shows the calibration results using the point based, line based, plane based and multi-feature based approaches to estimate LiDAR extrinsic calibration parameters $\Theta_{L}$. In plane based calibrated experiments, the standard deviation of $\rho$ and $\theta$ degrade with the increase of the vertical angle $\alpha$; the reason is that planar targets are greatly affected by the angle of incidence of the laser beam. As shown in Figure 5a and Figure $5 \mathrm{~b}$, the Max, Min, Mean values of the estimated standard deviation of $\rho$ and $\theta$ versus vertical angle $\alpha$ are $2.89 \mathrm{~mm}$, $1.73 \mathrm{~mm}, 2.32 \mathrm{~mm}$ and $0.027^{\circ}, 0.019^{\circ}, 0.023^{\circ}$. Similarly, The Max, Min, Mean values of the estimated standard deviation of $\rho$ and $\theta$ using line (cylinder) based method and point (sphere) based method are $2.57 \mathrm{~mm}, 1.82 \mathrm{~mm}, 2.03 \mathrm{~mm}$ and $0.023^{\circ}$, $0.018^{\circ}, 0.020^{\circ}$, and $2.09 \mathrm{~mm}, 1.76 \mathrm{~mm}, 1.90 \mathrm{~mm}$ and $0.022^{\circ}$, $0.017^{\circ}, 0.019^{\circ}$, respectively. In the multi-feature based calibration experiments, the sphere target, cylinder target and plane target were used to calibrate LiDAR extrinsic parameters $\Theta_{L}$, and the calibration results are following: the Max, Min, Mean values of the estimated standard deviation of $\rho$ and $\theta$ versus vertical angle $\alpha$ are $1.86 \mathrm{~mm}, 1.66 \mathrm{~mm}, 1.76 \mathrm{~mm}$ and $0.019^{\circ}, 0.016^{\circ}, 0.017^{\circ}$.

According to the above analysis, it can be concluded that the multi-feature based method can improve the calibration accuracy of LiDAR extrinsic parameters effectively.

\section{B. IMU Intrinsic Parameters Calibration Experiments}

After the LIDAR extrinsic parameters were fully calibrated using the multi-type geometric features, the rotation $T_{I}^{L}$ and the translation $R_{I}^{L}$ between LiDAR and IMU could be obtained based on Equation (3); then the $R_{I}^{L}$ and $T_{I}^{L}$ together with calibrated LiDAR extrinsic parameters were all applied to calibrate the IMU intrinsic parameters.

The LiDAR and IMU were fixed on the rotating platform. Suppose that the initial translation and rotation biases were computed by the total station instrument. The performances of IMU intrinsic parameter calibration were tested in various static modes.

During the static calibration mode, the turntable was rotated in different configurations, and the LiDAR-IMU system was placed in front of the targets for LiDAR extrinsic parameter calibration. After the LiDAR extrinsic calibration was completed, the translation $T_{L I}$, rotation $R_{L I}$, the nominal attitude angle values (heading, pitch, roll) provided by the high precision turntable were used to calibrate the IMU intrinsic parameters using the FIMLE method. The experimental results were presented in Figure 6. The horizontal axis represents the measurement time of the IMU and the vertical axis denotes the measured errors in heading, pitch and roll directions compared with the nominal accurate value provided by the high precision turntable. The black line shows the measurement errors of IMU before calibration, and the blue line shows the measurement errors of IMU after calibration using FIMLE. It can be observed through the comparisons in Figure 6a that the estimated errors in the heading orientation of the IMU were improved obviously after calibrated by FIMLE method, with the overall accuracy improvement being $21.86 \%$. Similar to the heading orientation, the overall accuracy improvements in pitch orientation (Figure $6 \mathrm{~b}$ ) and roll orientation (Figure 6c) are $17.63 \%$ and $13.36 \%$, respectively, after calibrated by FIMLE method.

After obtaining the IMU measurement results before and after calibration, Equation (31) was then used to estimate the IMU intrinsic parameters $\delta_{\alpha}, k_{\alpha}, \delta_{g} k_{g}$ according to Figure 6 and the acquired LiDAR extrinsic parameters. The results are shown in Table IV. The Table IV illustrates that the Multifeature based method can improve the overall accuracy in $\delta_{\alpha}$ by $27.78 \%, 20.69 \%$ and $18.45 \%$, respectively, compared with the plane based, Line based and Point based methods. Similarly, the overall accuracy improvements in $k_{\alpha}, \delta_{g}$ and $k_{g}$ are $34.72 \%, 27.68 \%, 25.39 \%$, and $40.23 \%, 24.76 \%, 22.89 \%$, and $33.33 \%, 22.81 \%, 20 \%$, respectively.

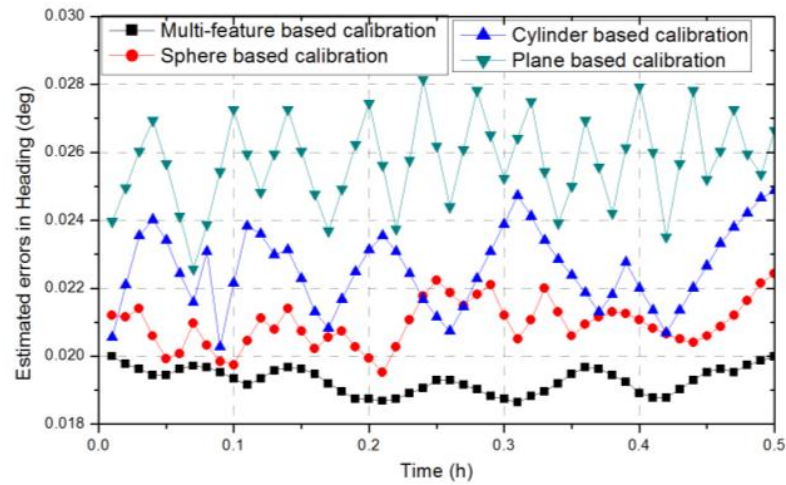

(a) The estimated errors in heading orientation

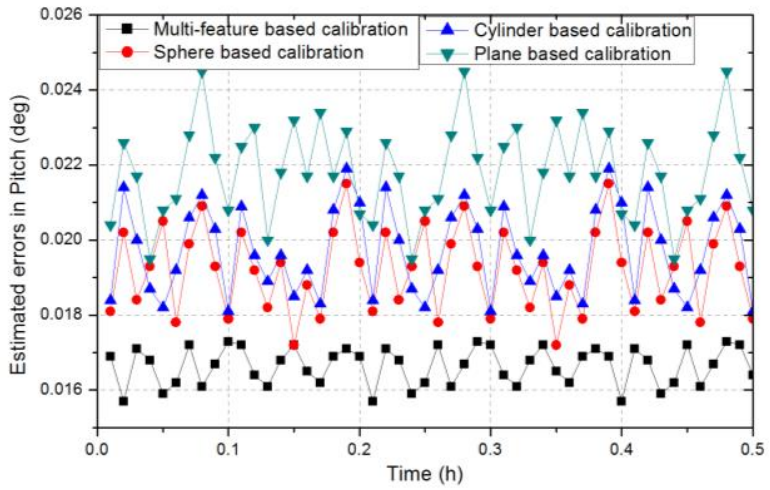

(b) The estimated error of the Pitch orientation

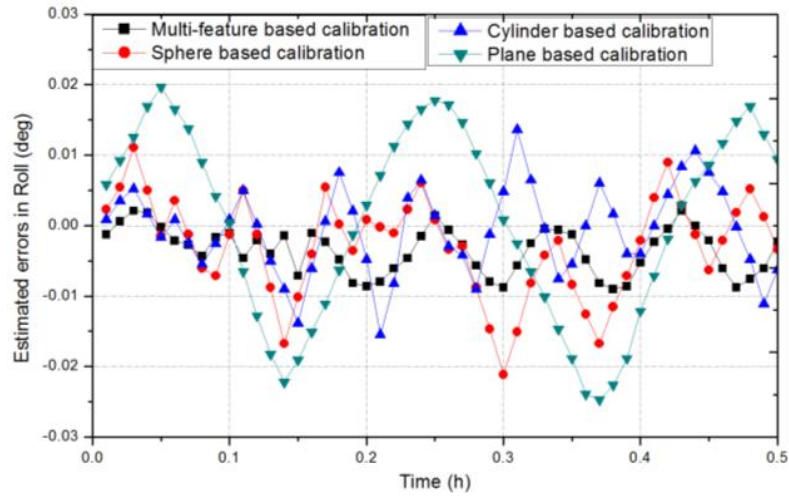

(c) The estimated error of the Roll orientation

Fig. 6. The calibration results of IMU intrinsic parameters 
TABLE IV

THE VALUES OF IMU INTRINSIC PARAMETERS AFTER CALIBRATION

\begin{tabular}{ccccc}
\hline $\begin{array}{c}\text { IMU intrinsic } \\
\text { parameters }\end{array}$ & $\begin{array}{c}\text { Plane } \\
\text { based } \\
\text { method }\end{array}$ & $\begin{array}{c}\text { Line } \\
\text { based } \\
\text { method }\end{array}$ & $\begin{array}{c}\text { Point } \\
\text { based } \\
\text { method }\end{array}$ & $\begin{array}{c}\text { Multi- } \\
\text { feature } \\
\text { based } \\
\text { method }\end{array}$ \\
\hline$\delta_{\alpha}\left(\mathrm{m} / \mathrm{s}^{2}\right)$ & 0.918 & 0.836 & 0.813 & 0.663 \\
$k_{\alpha}(\%)$ & 0.072 & 0.065 & 0.063 & 0.047 \\
$\delta_{g}(\mathrm{rad} / \mathrm{s})$ & 0.778 & 0.618 & 0.603 & 0.465 \\
$k_{g}(\%)$ & 0.066 & 0.057 & 0.055 & 0.044 \\
\hline
\end{tabular}

\section{VIII.CONCLUSIONS}

In this paper, a multi-feature based on-site calibration method was proposed for LiDAR-IMU system to improve the calibration flexibility. The primary contributions of this paper are that:

(1) This paper proposes a multi-feature based method for LiDAR-IMU on-site calibration without using any artificial targets or specific facility. This method combines the advantages of point, line and plane features to generate more accurate calibration results. It can be applied to calibrate any type of 3D or 2D LiDAR-IMU system. In addition, these features can be easily found in many indoor and outdoor scenes.

(2) The calibration process is easy to carry out. The LiDAR extrinsic parameters can be calibrated through estimating the LiDAR geometric features and solving the multi-feature optimization problem. The IMU intrinsic parameters can be calibrated using FIMLE. The relationships between LiDAR and IMU intrinsic calibration parameters can be obtained by the coordinate transformation.

(3) The experimental results show that the proposed method can improve the calibration accuracy of LiDAR extrinsic parameters and IMU intrinsic parameters.

Additionally, the developed calibration method can also support frequent and rapid calibration of the error parameters of the LiDAR-IMU system. Moreover, the proposed method can be potentially implemented for on-site real time calibration for vehicular and robotic, and it is very suitable to perform localization or navigation tasks in GPS-denied environments.

\section{REFERENCES}

[1] Wang Bo, Ren Qian, Deng Zhihong and Fu Mengyin, "A Self-Calibration Method for Non orthogonal Angles Between Gimbals of Rotational Inertial Navigation System," IEEE Trans. Ind. Electron., vol. 62, no.4, pp. 2353-2362, 2015.

[2] Nikolic Janosch, Burri Michael, Gilitschenski Igor, Nieto Juan and Siegwart Roland, "Non Parametric Extrinsic and Intrinsic Calibration of Visual-Inertial Sensor Systems," IEEE Sensors Journal, v 16, n 13, pp 5433-5443, 2016.

[3] Huang Lili and Barth Matthew, "A novel multi-planar LIDAR and computer vision calibration procedure using 2D patterns for automated navigation", IEEE Intelligent Vehicles Symposium. pp 117-122, 2009.

[4] Song Haryong, Choi Wonsub and Kim Haedong, "Robust Vision-Based Relative-Localization Approach Using an RGB-Depth Camera and LiDAR Sensor Fusion," IEEE Trans. Ind.Electron., vol. 63, no. 6, pp. 3725-3736, 2016.
[5] Liu, W.I., "Novel method for sphere target detection and center estimation from mobile terrestrial laser scanner data," Measurement, v 137, p 617623, 2019

[6] Aghili Farhad and Su Chun-Yi, "Robust relative navigation by integration of ICP and adaptive Kalman filter using laser scanner and IMU," IEEE Trans. Ind.Electron., vol. 63, no.4, pp. 2015-2026, 2016.

[7] Kelly Jonathan, Roy Nicholas and Sukhatme Gaurav S, "Determining the time delay between inertial and visual sensor measurements", IEEE Transactions on Robotics, v 30, n 6, pp 1514-1523, 2014.

[8] Marcelo Pereira, Vitor Santos and Paulo Dias, "Automatic calibration of multiple LIDAR sensors using a moving sphere as target," Advances in Intelligent Systems and Computing, v 417, p 477-489, 2016.

[9] Chen Shoubin, Liu Jingbin, Wu Teng, Huang Wenchao, Liu Keke, Yin Deyu, Liang Xinlian, Hyyppä Juha and Chen Ruizhi, "Extrinsic calibration of 2D laser rangefinders based on a mobile sphere," Remote Sensing, v 10, n 8, 2018.

[10] Kang Jaehyeon and Doh Nakju Lett, "Full-DOF Calibration of a Rotating 2-D LIDAR with a Simple Plane Measurement," IEEE Transactions on Robotics, v 32, n 5, p 1245-1263, 2016.

[11] Atanacio-Jiménez Gerardo; González-Barbosa José-Joel; Hurtado Ramos Juan B; Ornelas-Rodríguez Francisco J; Jiménez-Hernández Hugo; García-Ramirez Teresa and González-Barbosa Ricardo, "LIDAR velodyne HDL-64E calibration using pattern planes," International Journal of Advanced Robotic Systems, v 8, n 5, p 70-82, 2011.

[12] Huang Po-Sen, Hong Wen-Bin, Chien Hsiang-Jen and Chen Chia-Yen, "Extrinsic calibration of a multi-beam LiDAR system with improved intrinsic laser parameters using v-shaped planes and infrared images," IEEE 11th IVMSP Workshop: 3D Image/Video Technologies and Applications, pp 1-5, Seoul, South Korea, 2013.

[13] Shang Erke, An Xiangjing, Shi Meiping, Meng Deyuan, Li Jian and Wu Tao, "An Efficient Calibration Approach for Arbitrary Equipped 3-D LiDAR Based on an Orthogonal Normal Vector Pair," Journal of Intelligent and Robotic Systems: Theory and Applications, v 79, n 1, p 21-36, 2015.

[14] Zhou Lipu, "A new minimal solution for the extrinsic calibration of a 2D LIDAR and a camera using three plane-line correspondences," IEEE Sensors Journal, v 14, n 2, pp 442-454, 2013.

[15] Chan Ting On and Lichti Derek D, "Automatic In Situ Calibration of a Spinning Beam LiDAR System in Static and Kinematic Modes," Remote Sensing, v 7, n 8, p 10480-10500, 2015.

[16] Liu W.I. and Li Yunwang, "Error modeling and extrinsic-intrinsic calibration for LiDAR-IMU system based on cone-cylinder features," Robotics and Autonomous Systems, v 114, p 124-133, 2019.

[17] Gaurav Pandey, James R. McBride and Silvio Savarese, "Automatic Extrinsic Calibration of Vision and Lidar by Maximizing Mutual Information," Journal of Field Robotics, v 32, n 5, 696-722, 2015.

[18] Yoonsu Park, Seokmin Yun and Chee Sun Won, "Calibration between color camera and 3D LIDAR instruments with a polygonal planar board," Sensors (Switzerland), v 14, n 3, pp 5333-5353, 2014.

[19] Chen C Y, Chien H J, "Geometric calibration of a multi-layer LiDAR system and image sensors using plane-based implicit laser parameters for textured 3-D depth reconstruction", Journal of Visual Communication \& Image Representation, v 25, n 4, pp 659-669, 2014.

[20] Liu, W.I., "Novel method for sphere target detection and center estimation from mobile terrestrial laser scanner data," Measurement, v 137, p 617623, April 2019.

[21] Chen C Y, Chien H J, "On-Site Sensor Recalibration of a Spinning MultiBeam LiDAR System Using Automatically-Detected Planar Targets“, Sensors, v 12, n 10, pp 13736-13752, 2012.

[22] Zhenfei Yang and Shaojie Shen, "Monocular Visual-Inertial State Estimation With Online Initialization and Camera-IMU Extrinsic Calibration," IEEE Transactions on Automation Science \& Engineering, v 14, n 1, p 39-51, 2017.

[23] Liu Wanli, "Lidar-imu time delay calibration based on iterative closest point and iterated sigma point kalman filter," Sensors (Switzerland), v 17, n 3, pp. 539, 2017.

[24] Clement Deymier, Celine Teuliere and Thierry Chateau, "Self-calibration of a vehicle's acquisition system with cameras, IMU and 3D LiDAR", Traitement Du Signal, v 32, pp 121-145, 2015.

[25] Ting On Chan, Derek D. Lichti and David Belton, "A rigorous cylinderbased self-calibration approach for terrestrial laser scanners," Isprs Journal of Photogrammetry \& Remote Sensing, v 99, pp 84-998, 2015.

[26] Liu Yong, Xiong Rong, Wang Yue, Huang Hong, Xie Xiaojia, Liu, Xiaofeng and Zhang Gaoming, "Stereo Visual-Inertial Odometry with 
Multiple Kalman Filters Ensemble,” IEEE Trans. Ind. Electron., vol. 63, no. 10 , pp. 6205-6216, 2016.

[27] Shang Erke, An Xiangjing and Shi Meiping, "An Efficient Calibration Approach for Arbitrary Equipped 3-D LiDAR Based on an Orthogonal Normal Vector Pair", Journal of Intelligent \& Robotic Systems, v 79, n 1, pp 21-36, 2015.

[28] Jonathan Kelly and Gaurav S Sukhatme, "Visual-Inertial Sensor Fusion: Localization, Mapping and Sensor-to-Sensor Self-calibration", International Journal of Robotics Research, v 30, n 1, pp 56-79, 2011.

[29] Pandey, Gaurav, James R. McBride, Silvio Savarese and Ryan M. Eustice, "Automatic Extrinsic Calibration of Vision and Lidar by Maximizing Mutual Information", Journal of Field Robotics, v 32, n 5, pp 696-722, 2014.

[30] He Mengwen, Zhao Huijing, Davoine Franck, Cui Jinshi and Zha Hongbin, "Pairwise LIDAR calibration using multi-type 3D geometric features in natural scene," IEEE International Conference on Intelligent Robots and Systems, pp 1828-1835, Tokyo, Japan, 2013.

[31] Velodyne HDL-16E User's Manual. 2015. Available online: http://velodynelidar.com/lidar/ products/manual/63-9113\%20HDL32E\%20manual_Rev\%20E_NOV2012.pdf.

[32] Jeff Ferguson, "Calibration of Deterministic IMU Errors," M.S. thesis, Embry-Riddle Aeronautical University, Daytona Beach. USA, 2015.

[33] Derya Unsal , Kerim Demirbas, "Estimation of deterministic and stochastic IMU error parameters", Position Location and Navigation Symposium. IEEE, pp862-868, 2012. 